\title{
Immunogold localisation of laminin in normal and exfoliative iris
}

\author{
A G Konstas, G E Marshall, W R Lee
}

University of Glasgow, Western Infirmary, Glasgow Department of Ophthalmology A G Konstas

Department of Pathology G E Marshall W R Lee

Correspondence to: Dr A G Konstas, Department of Ophthalmology, University of Glasgow, Western Infirmary, Glasgow G11 6NT Accepted for publication 29 March 1990
Abstract

Immunoelectron microscopic studies of exfoliative iris tissue (seven specimens) revealed the presence of laminin in the fibrillar component of exfoliation material. The immunogold label was uniformly distributed on the exfoliation fibres. Deposition of laminin labelled exfoliation material in the dilator muscle was a noteworthy feature, as was an apparent depletion of laminin in the basement membranes of ostensibly unaffected vessels. In control iris tissue (five enucleated eyes) laminin was identified in the basement membrane round vascular contractile cells, but not beneath the endothelium.

Exfoliation syndrome, alternatively referred to as pseudoexfoliation syndrome, is an intriguing

TABLE I Clinical details: normal iris cases

\begin{tabular}{llll}
\hline Case no & Age & Sex & Indication for enucleation \\
\hline 1 & 28 & M & Choroidal melanoma \\
2 & 63 & F & Choroidal melanoma \\
3 & 65 & M & Choroidal melanoma \\
4 & 69 & M & Choroidal melanoma \\
5 & 81 & F & Secondary glaucoma \\
\hline
\end{tabular}

TABLE II Clinical summary of patients with exfoliation

\begin{tabular}{|c|c|c|c|c|c|}
\hline $\begin{array}{l}\text { Case } \\
\text { no }\end{array}$ & Age & Sex & $\begin{array}{l}\text { Clinical } \\
\text { presentation }\end{array}$ & $\begin{array}{l}\text { Other clinical } \\
\text { findings }\end{array}$ & $\begin{array}{l}\text { Indication } \\
\text { for surgery }\end{array}$ \\
\hline 1 & 82 & $\mathbf{M}$ & $\begin{array}{l}\text { Exf syndrome } \\
\text { Normal IOP } \\
\text { C/D ratio }=0.5\end{array}$ & $\begin{array}{l}\text { Iridodonesis } \\
\text { Partial lens } \\
\text { subluxation }\end{array}$ & $\begin{array}{l}\text { Cataract } \\
\text { extraction }\end{array}$ \\
\hline 2 & 69 & $\mathbf{M}$ & $\begin{array}{l}\text { Exf glaucoma } \\
\text { IOP }=32 \mathrm{mmHg} \\
\text { VF loss stage I } \\
\mathrm{C} / \mathrm{D} \text { ratio }=0.6\end{array}$ & $\begin{array}{l}\text { Iris pigm } \\
\text { Angle Pigm 3+ } \\
\text { SPL }\end{array}$ & $\begin{array}{l}\text { Failed } \\
\text { medical Rx }\end{array}$ \\
\hline 3 & 79 & $\mathbf{F}$ & $\begin{array}{l}\text { Exf glaucoma } \\
\text { IOP }=34 \mathrm{mmHg} \\
\text { VF loss stage II } \\
\text { C/D ratio }=0.6\end{array}$ & $\begin{array}{l}\text { Angle pigm 3+ } \\
\text { SPL }\end{array}$ & $\begin{array}{l}\text { Failed } \\
\text { medical Rx }\end{array}$ \\
\hline 4 & 69 & $\mathbf{M}$ & $\begin{array}{l}\text { Exf glaucoma } \\
\text { IOP }=24 \mathrm{mmHg} \\
\text { VF loss stage III } \\
\mathrm{C} / \mathrm{D} \text { ratio }=0.6\end{array}$ & $\begin{array}{l}\text { Corneal pigm } \\
\text { Angle pigm 3+ } \\
\text { SPL }\end{array}$ & $\begin{array}{l}\text { Failed } \\
\text { medical Rx }\end{array}$ \\
\hline 5 & 75 & $\mathbf{M}$ & $\begin{array}{l}\text { Exf glaucoma } \\
\text { IOP }=50 \mathrm{mmHg} \\
\text { VF loss stage III } \\
\mathrm{C} / \mathrm{D} \text { ratio }=0.6\end{array}$ & $\begin{array}{l}\text { Iridodonesis } \\
\text { Angle pigm 4+ } \\
\text { SPL }\end{array}$ & $\begin{array}{l}\text { Poor } \\
\text { compliance }\end{array}$ \\
\hline 6 & 68 & $\mathbf{M}$ & $\begin{array}{l}\text { Exf glaucoma } \\
\text { IOP }=50 \mathrm{mmHg} \\
\text { VF loss stage IV } \\
\text { C } / \mathrm{D} \text { ratio }=0.7\end{array}$ & $\begin{array}{l}\text { RAPD } \\
\text { Angle pigm 2+ }\end{array}$ & $\begin{array}{l}\text { Early } \\
\text { trabeculectomy }\end{array}$ \\
\hline 7 & 65 & $\mathbf{M}$ & $\begin{array}{l}\text { Exf glaucoma } \\
\text { IOP }=34 \mathrm{mmHg} \\
\text { VF loss stage IV } \\
\mathrm{C} / \mathrm{D} \text { ratio }=0.8\end{array}$ & $\begin{array}{l}\text { Exf on cornea } \\
\text { Iris pigm } \\
\text { Angle pigm 3+ } \\
\text { Phakodonesis }\end{array}$ & $\begin{array}{l}\text { Early } \\
\text { trabeculectomy }\end{array}$ \\
\hline
\end{tabular}

Exf $=$ exfoliation. $\mathrm{VF}=$ visual field. $\mathrm{C} / \mathrm{D}=$ cup to disc. $\mathrm{Pigm}=$ pigmentation. $\mathrm{SPL}=$ Sampaolesi's line present. $\mathrm{RAPD}=$ relative afferent pupiliary defect. $\mathrm{Rx}=$ treatment . age-related ocular disorder characterised by the production and deposition of an abnormal protein in the anterior segment of the eye. The disease is of major clinical significance, since it is intimateiy associated with glaucoma. ${ }^{12}$ Intensive studies have not yet revealed the precise nature of the exfoliation material.

By light microscopy the appearance and distribution of exfoliation material are well documented. In brief, eosinophilic clumps of bushlike material are deposited on the ciliary processes, the zonular fibres, the surfaces of the lens, and the iris, and within the trabecular meshwork and stroma of the conjunctiva. ${ }^{3}$ Electron microscopy shows exfoliation material to consist of fibres formed from filamentous subunits, with a varied periodicity $(17-56 \mathrm{~nm})$ and embedded in an interfibrillar matrix. ${ }^{34}$ It is generally accepted that exfoliation material is composed of a variety of biochemical constituents. ${ }^{5}$ Histochemical staining was obtained for proteoglycans $^{6}$ and glycoproteins, ${ }^{7}$ and similarities were shown between exfoliation material and the ocular zonular fibres, ${ }^{8}$ oxytalan, ${ }^{9}$ and constitutents of the elastic system. ${ }^{10-12}$

It has long been speculated that the material may be abnormal basement membrane synthesised at multiple sites by defective aging cells. ${ }^{13}$ Since basement membranes contain collagenous and non-collagenous components, disordered synthesis or degradation of noncollagenous components may be a significant feature of the disorder. ${ }^{14}$

In normal tissue one non-collagenous component, namely laminin, has been identified by established immunocytochemical techniques. ${ }^{15}$ ${ }_{16}$ We therefore decided to investigate the presence of laminin in exfoliation material. Exfoliative iris tissue was used in this study on account of the early involvement of the iris in the disease process $^{17}$ and the great abundance of exfoliation material present therein. ${ }^{18}$ As laminin distribution has not been described in the normal iris, we included an examination of an adequate sample of normal iris tissue as an integral part of this investigation.

Patients and methods

Normal iris tissue was obtained from freshly enucleated eyes of four patients suffering from choroidal melanoma and one patient with absolute glaucoma (Table I). Representative blocks were dissected from the peripheral part of 
the iris, and control tissue for laminin was taken from the central part of the cornea. The iris specimens were ostensibly normal on microscopic examination of toluidine blue stained LR white (London resin white) semithin sections.

Exfoliative iris tissue was obtained from seven peripheral iridectomies performed during six trabeculectomies and one intracapsular cataract extraction. Table II provides the clinical details of these patients. The visual field loss was graded from I to V stages by the classification described by Jay and Murray. ${ }^{19}$ Five of the patients were examined by one of us (AGK) before the operation. The other two patients had been examined by their ophthalmologist in a sister hospital. All the surgical specimens were processed in an identical manner.

\section{FIXATION AND PLASTIC EMBEDDING}

The specimens were immersed immediately after enucleation or iridectomy in a freshly made fixative solution of $4 \%$ paraformaldehyde plus 0-1\% glutaraldehyde in $0 \cdot 1 \mathrm{M}$ Sörensen's phosphate buffer or cacodylate buffer at $\mathrm{pH} 7 \cdot 2$. After 10 minutes the enucleated eyes were opened to allow for rapid penetration of the fixative into the globe. Fixation was continued for two hours at room temperature. After fixation the blocks were washed three times in buffer $(5 \mathrm{~min})$ and processed for LR white embedding, the details of which have been described. ${ }^{20}$

\section{ANTIBODIES}

Polyvalent rabbit antibodies against laminin were supplied by Heyl (Berlin, West Germany) and had been raised in rabbits by multiple injections of human laminin. The serum was tested by the supplier with the following immunological assays: ELISA, immunoblot, and immunohistology (fluorescence, APAAP).

\section{IMMUNOCYTOCHEMISTRY}

Ultrathin sections (silver-gold) were cut on an LKB Ultrotome Nova(Cambridge Instruments), mounted on 200 mesh nickel grids, and left to dry for 10 minutes before rehydrating on drops of distilled water. The antibody dilutions in trometamol (Tris) buffer plus $1 \%$ bovine serum albumin (BSA), determined by previous experimental work, were 1:20-1:40. Incubation with the laminin primary antibody was performed at room temperature for two hours. Immunogold labelling was performed with a 1:40 dilution of goat anti-rabbit gold conjugate $(10 \mathrm{~nm})$, which was supplied by Biocell Laboratories, Reading, UK. The precise immunocytochemical procedure is described elsewhere. ${ }^{1520}$

\section{CONTROLS}

Normal rabbit serum negative controls (Sigma) were concurrently conducted at the same dilutions. Omission of the primary antibody was also used as a negative control. Human cornea, fixed in $4 \%$ paraformaldehyde for two hours and processed in an identical manner to the iris specimens examined, was used as a positive control for laminin. ${ }^{15}$

\section{Results}

NORMAL IRIS

In all five iris specimens laminin was specifically localised to the basement membranes within the walls of blood vessels and to the extracellular matrix in the dilator muscle region. The specific features are as follows:

Vasculature. The label was most intense on the matrix surrounding the myocytes of the larger iris vessels (Fig 1A). Labelling for laminin was prominent in large and medium sized vessels and greatly reduced or absent in small vessels. When present in small vessels, immunolabelling was seen to be more intense on the outer side of pericytes. Of the five cases studied the highest intensity of labelling around small vessels was observed in the youngest individual (see Table I): in this particular specimen laminin was demonstrated throughout the whole of the iris vasculature. In contrast, laminin was markedly reduced in iris vessels from the oldest patient.

Pigment epithelium. Laminin was localised to the extracellular matrix in which the basal muscular portion of the anterior iris pigment epithelium was embedded (Fig 1B). Immunolabelling was mainly concentrated on dense bands of filaments within an amorphous granular substance filling the intercellular spaces. Labelling intensity increased in proportion to the quantity and density of the filamentous bands and was mainly restricted to those electron dense areas in all normal iris specimens studied. Laminin was also demonstrated on the basement membrane of the anterior iris pigment epithelium. The thin basal membrane of the posterior pigment epithelium of the iris showed weak but specific labelling.

Of particular interest was the strong signal associated with iris fibrocytes which form the boundary between the dilator muscle region and iris stroma. Labelling was intense on filamentous bodies adjacent to these fibrocytes (Fig 1C). Among the five normal iris specimens studied the highest density of immunogold particles in the dilator muscle region was in the youngest patient.

Stroma and anterior border layer. Gold particles were absent from the iris stroma and associated cells (fibrocytes, melanocytes, and mast cells). However, a discrete localised label was present on small clusters of filaments associated with multilayered fibrocytes of the anterior border layer. Such labelling was generally absent from the outermost layer of fibrocytes.

\section{EXFOLIATIVE IRIS}

Exfoliation material. Intense labelling for laminin was observed over areas where exfoliation material was present within the iris. This finding was consistent in all seven exfoliative iris cases. Labelling was regarded as extremely specific on the basis of the absence of gold particles from the nuclei, cell cytoplasm, and lumina of blood vessels. Better antigenic preservation was 


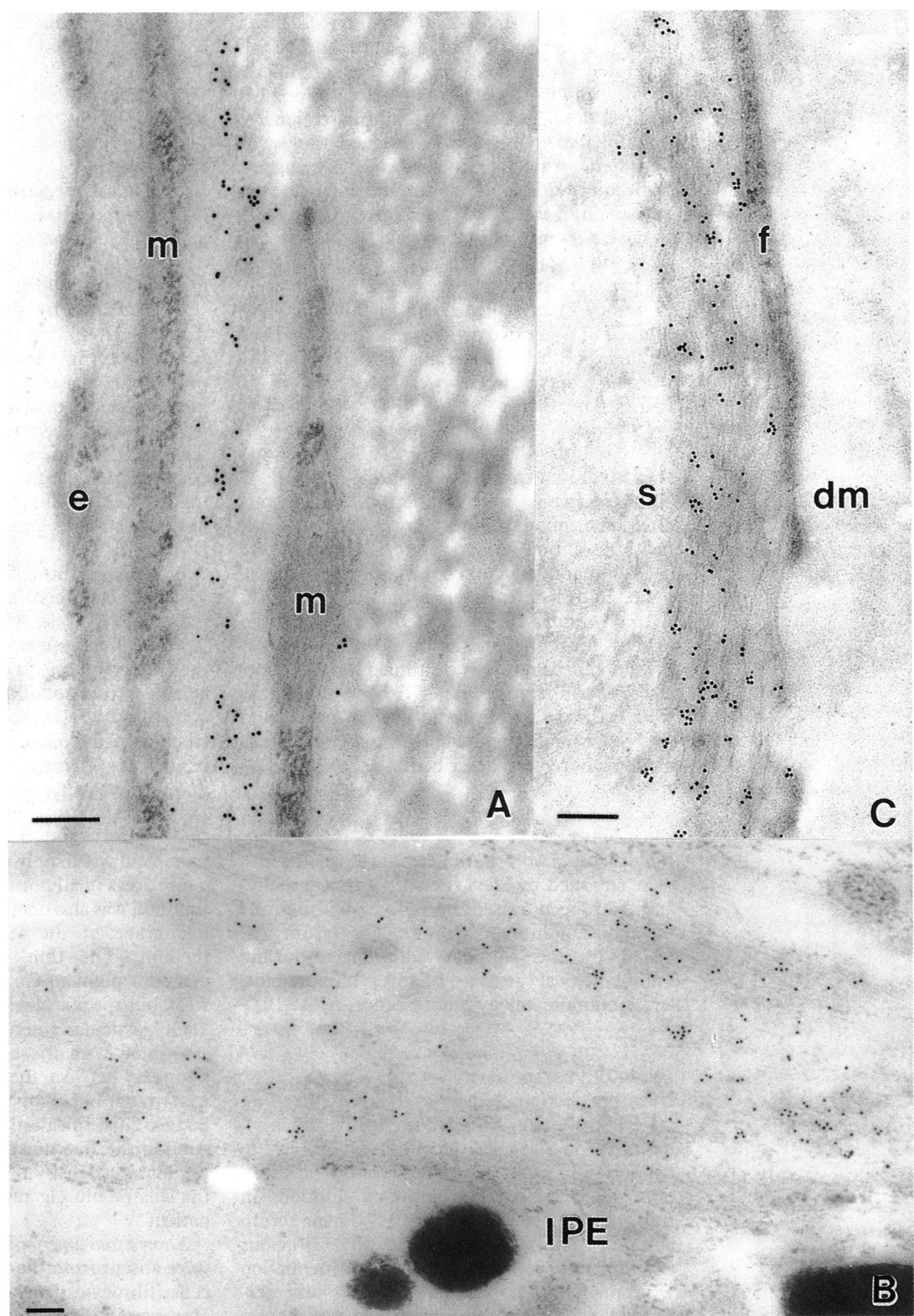

Figure 1: Labelling for laminin in the extracellular matrix of the normal iris. A: In the wall of a vessel there is a positive signal in the fibrillar matrix between two myocytes $(m)$, but not in the basement membrane of the endothelium $(e)$. B: In the matrix anterior to the anterior iris pigment epithelium (IPE) laminin is localised to the clumps of filamentous electron dense material. $C$ : The parallel filamentous bundles adjacent to the stromal fibrocytes (f) exhibit a strong signal for laminin. (dm): Dilator muscle region. (s): Iris stroma. (Bar $=200 \mathrm{~nm}$.)

achieved in formaldehyde fixed tissue, but this was at some expense of ultrastructural preservation. In glutaraldehyde fixed tissue immunolabelling was less intense but highly specific. Recognition of the exfoliation material was relatively easy in $\mathrm{LR}$ white processed tissue. This was due to a difference in tissue contrast in comparison with conventionally processed tissue, which resulted in greater detail in the extracellular matrix components. In addition it was found that larger amounts of extracellular matrix material were retained with the LR White embedding procedure. Laminin was present predominantly in the exfoliation fibres, but weak labelling was also observed on the electronlucent interfibrillar matrix (Fig 2A, B).

In four of the cases, vegetations consisting of laminin labelled exfoliation material were seen to project into the anterior chamber from the anterior border layer (Fig 2A). Within an 


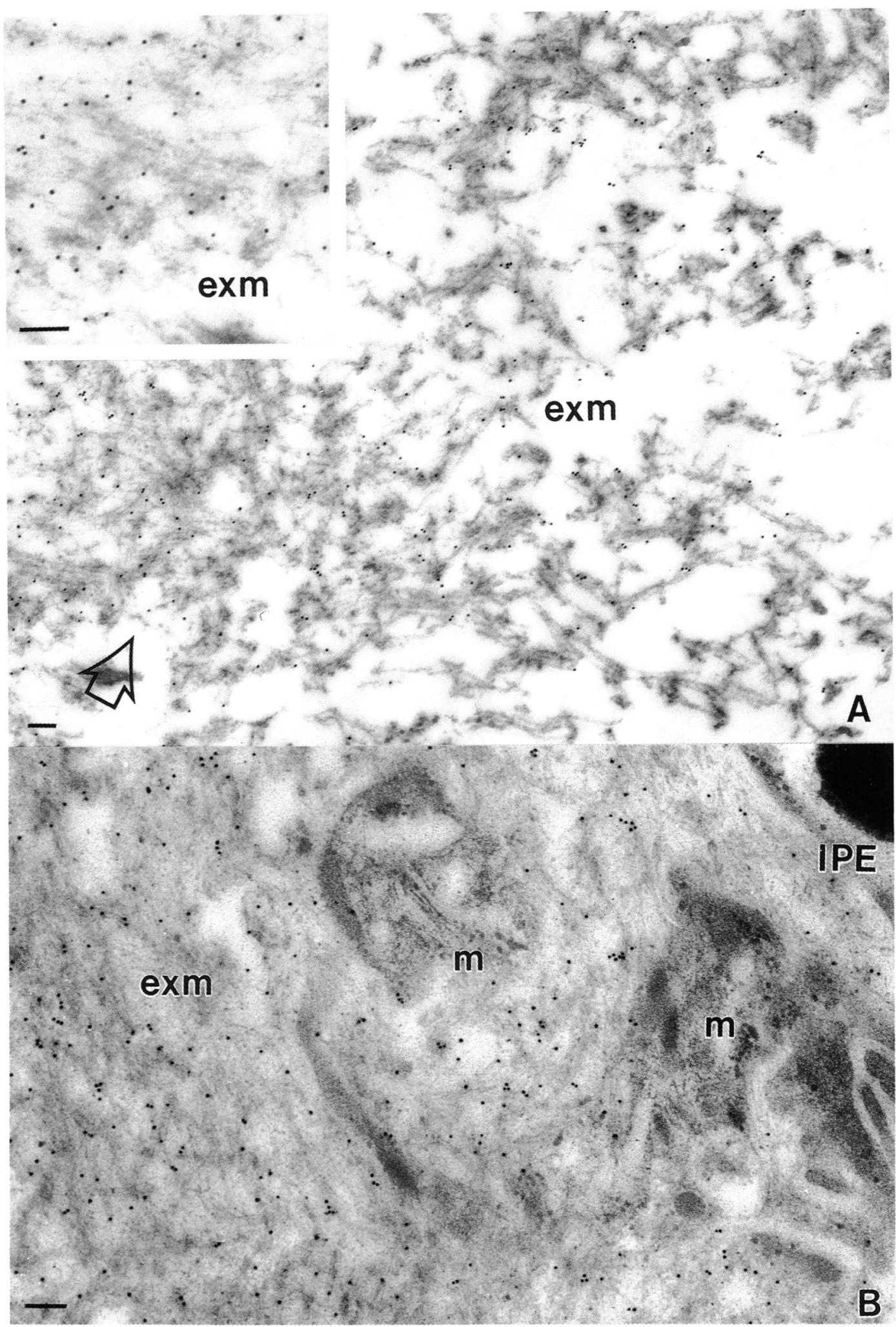

Figure 2: A: Positive laminin labelling in the loosely arranged exfoliation material (exm) in a vegetation on the anterior iris surface. The labelling is predominantly on the fibrillar component (see inset for high magnification of area marked with an arrow). A similar labelling pattern is seen in the more compact band of exfoliation material adjacent to the iris pigment epithelium $(I P E)$ and around the processes of the dilator muscle $(m) .(B a r=200 \mathrm{~nm}$.

individual case the intensity of immunogold localisation was proportional to the density of the exfoliative deposits. The highest labelling intensity was observed on dense deposits in the dilator muscle region (Fig $2 \mathrm{~B}$ ) and round large iris vessels.

Vasculature. In the exfoliative iris the walls of some blood vessels were found to be free from deposits of exfoliation material. Rather surprisingly, in such exfoliation-free vessels, base- ment membrane labelling for laminin was markedly reduced or absent in comparison with normal iris vessels.

In exfoliative vessels (that is, vessels associated with exfoliation material) immunolabelling was predominantly localised to the exfoliation material (Fig 3A, B, C). In all vessels in which clumps of exfoliation material were observed a thin label-free zone was noted corresponding to the lamina lucida of endothelial cell basement 


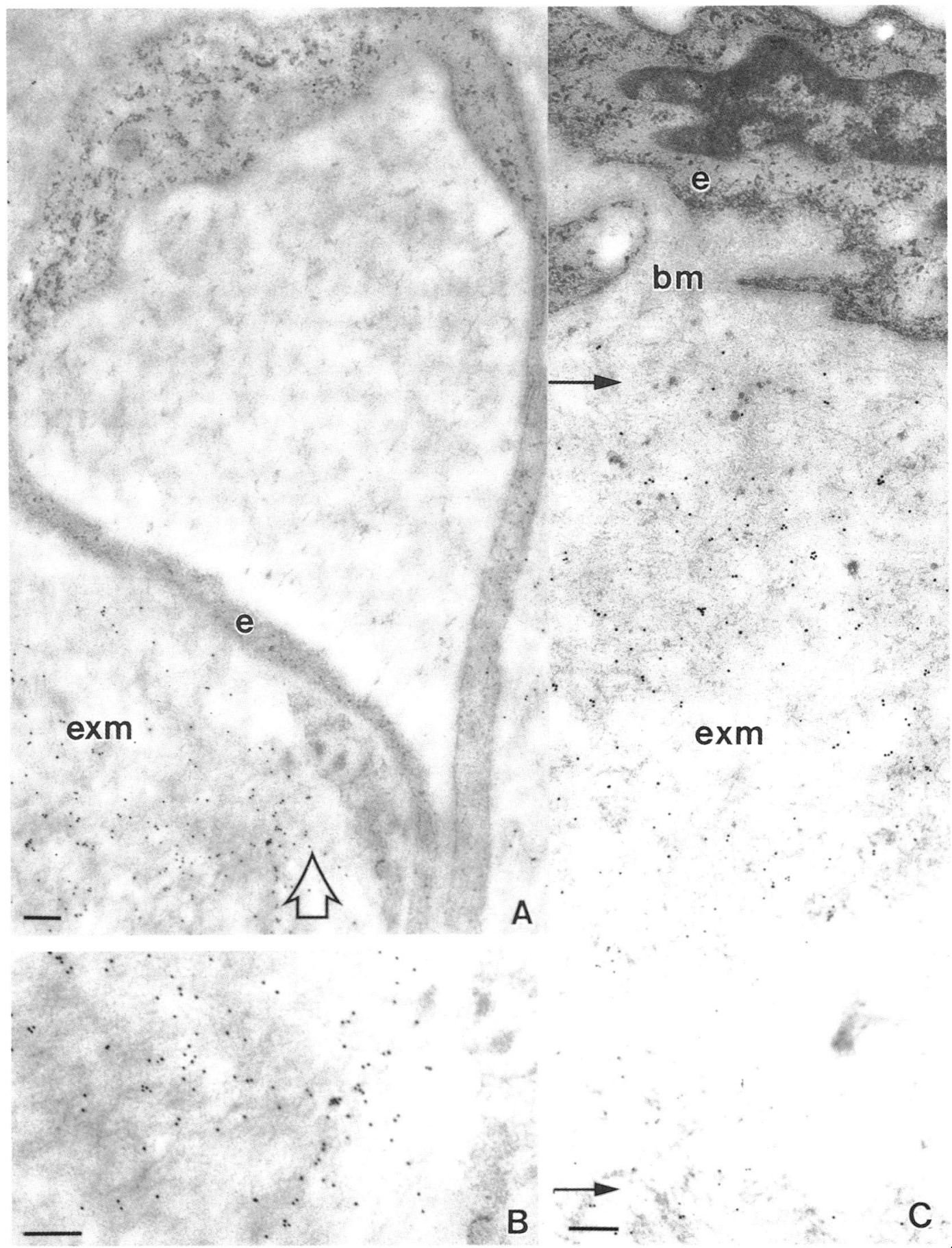

Figure 3: Exfoliation material labelled with laminin in the walls of iris vessels. A: The endothelium (e) appears atrophic and the exfoliation material (exm) had a poorly defined fibrillar component because the fixative was glutaraldehyde-free. $B:$ The area marked with an arrow is shown at high magnification. C: The total extent (between arrows) of the exfoliation material (exm) deposition around a vessel is shown; glutaraldehyde in the fixative intensifies the fibrillar component. Note the absence of immunolabelling from the basement membrane $(\mathrm{bm})$ of the endothelium $(e) .(B a r=200 \mathrm{~nm}$.)

membrane. The adjacent lamina densa was often obscured by exfoliation material, which was strongly labelled for laminin (Fig 3). The clumps of exfoliation material often extended from within the walls of the iris vessels into the iris stroma. In many of the exfoliative vessels containing exfoliation material the endothelial cells were attenuated and the myocytes were atrophic (Fig 3A). This was particularly evident in advanced cases of exfoliation glaucoma.

Pigment epithelium. We were intrigued with the association between exfoliation material and the dilator muscle (Fig 2B). In four of the specimens exfoliation material had infiltrated the basal muscular portion of the anterior iris pigment epithelium, causing a marked disturbance in the architecture of this layer. Laminin was again mainly localised in the exfoliation fibres. In case 7, where exfoliation material had infiltrated wide areas of the dilator muscle region (Fig 2B), laminin labelling on the deposits was intense. Exfoliation material frequently surrounded the muscular processes of the anterior iris pigment epithelium and was observed between the intact walls of epithelial cells, but did not penetrate the cell membrane. In no specimen was exfoliation material noted between the two layers of the pigment epithelium, nor was it seen on the basal 


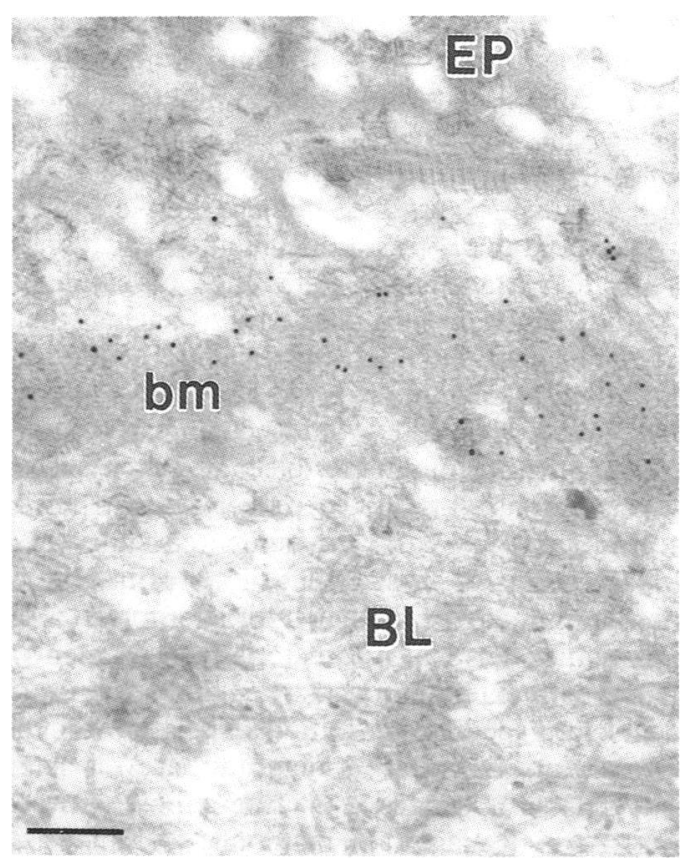

Figure 4: Example of positive corneal control. Laminin is localised in the basement membrane $(\mathrm{bm})$ beneath the epithelium $(E P)$ of the cornea. Note the absence of labelling in Bowman's layer $(B L)(B a r=200 \mathrm{~nm}$. $)$

surface of the posterior layer of the pigment epithelium. The latter finding may have been due to the loss of exfoliation material during surgical handling of the tissue.

With regard to the positive corneal control employed in this study, laminin was demonstrated in the basement membrane of the corneal epithelium (Fig 4). Our findings for laminin in the human cornea are in agreement with previously described immunohistochemical results for human cornea at the light microscopical level. ${ }^{21}$

\section{Discussion}

Laminin is a large multidomain glycoprotein which together with collagen type IV and heparan sulphate proteoglycan (HSPG) comprises most of the protein found in basement membranes. ${ }^{22}$ The extracellular matrix possesses important regulatory properties governing cell behaviour. ${ }^{23}$ More specifically, basement membranes are specialised sheets of extracellular matrix directly involved in a number of important biological processes, such as cell adhesion and orientation. ${ }^{24}$ Moreover, it is thought that individual components within basement membranes are responsible for particular functions. Thus collagen type IV forms network structures which provide the structural scaffold on to which the other components are assembled, ${ }^{25}$ whereas HSPG maintains the filtration properties of basement membranes. ${ }^{22}$

Laminin promotes the structural integrity of basement membranes through specific binding with collagen type IV and HSPG, but of greater importance is its functional contribution to basement membranes. It has been shown that laminin promotes cell adhesion, migration, and growth and has an active role in tissue restoration. ${ }^{26} 27$ These functions are associated with specific functional domains within the laminin molecule. For example, the mitogenic and cell attachment activities are two distinct functions controlled by separate laminin domains. ${ }^{28}$

It is surprising that so little attention has been paid to the distribution of laminin in the normal iris considering that the iris has many unique structural and functional properties. This information is obviously necessary in order the better to comprehend the functional role of laminin in iris.

It was of interest to note the distribution of laminin in the normal iris vasculature. Labelling for laminin was restricted principally to the extracellular matrix enclosing the myocytes within the walls of the larger iris vessels. A similar pattern was also observed in smaller vessels when labelling was observed round contractile cells but not beneath the vascular endothelium (Fig 1A). The same localisation pattern for laminin was reported in a recent immunocytochemical study of cat retinal vessels. ${ }^{29}$ This pattern of laminin distribution may indicate that within the iris vasculature the synthesis of laminin is a responsibility of the myocytes and pericytes rather than the endothelial cells. Our conclusion is based on the assumption that cells attached to basement membranes secrete most if not all the extracellular matrix components present within that basement membrane.

By contrast, in a previous study ${ }^{15}$ we have shown that there was more intense labelling for collagen type IV in the subendothelial basement membrane of iris vessels in comparison with the matrix round vessel supporting cells. Consequently we consider that in iris vessels endothelial cells assemble primarily collagen type IV while laminin is mainly produced by myoepithelial cells and pericytes. This is important, as differences in the production and utilisation of laminin may contribute to variations in cell behaviour. It is relevant that a recent in-vitro study demonstrated that aortic endothelial cell adhesion is preferentially promoted by collagen type IV in comparison with laminin. ${ }^{27}$

It has recently been emphasised that the chemical composition of basement membranes may vary according to the demands of particular tissues. ${ }^{22}$ The observation that in the normal iris laminin seems to be mainly present in the walls of thicker vessels indicates heterogeneity in the components of thick and thin basement membranes within the iris. Our findings are in accord with an immunogold quantitative study performed on a variety of animal basement membranes. ${ }^{30}$

With reference to the label exhibited for laminin in the matrix in which the dilator muscle is embedded, we suggest that in this instance laminin may be acting as an adhesive factor. Its presence in the matrix may be instrumental for the orderly contraction of the dilator muscle. Therefore changes in its distribution within this matrix could conceivably interfere with the dilatation of the iris.

The intense labelling associated with the filamentous bundles bordering iris stromal fibrocytes is more difficult to interpret, as the functional role of these cells remains unclear. However, a recent ultrastructural study has demonstrated a layer of iris fibrocytes separating the dilator muscle cells from the iris stroma, and 
it postulated that these fibrocytes played a role in the innervation of the dilator muscle. ${ }^{31}$

There is accumulating evidence that alterations in the interaction between cells and their matrices can precede and may eventually result in ocular tissue malfunction..$^{213233}$

It is conceivable that during the aging process quantitative alterations of specific extracellular matrix components occur within ocular tissues and may result in functional impairment. Such a process could account for the reduction of laminin associated with aging in our material. Nevertheless, the effect of aging on the quantity of laminin can be confirmed only with the use of an immunogold quantitation technique.

To the best of our knowledge this is the first investigation that has shown laminin to be an integral component of exfoliation material. An intense specific label associated with exfoliation aggregates was obtained in all seven specimens. Labelling was principally present on the exfoliation fibres, with a low density of label associated with the interfibrillar matrix. At high magnification immunolabelling was shown to be evenly distrubted over the exfoliation fibres. Moreover, the labelling density increased with increasing density of exfoliation deposits. Therefore our results demonstrate that laminin constitutes an integral component of the exfoliation fibres and to a less extent to that of the interfibrillar matrix.

We were intrigued to find that labelling was markedly reduced in the basement membrane of exfoliation-free iris vessels. Within the vessels containing exfoliation material the immunogold label was predominantly localised to the exfoliation material. However, it was not possible to be certain that laminin was depleted from basement membranes in exfoliation-positive iris vessels, since exfoliation material was intermingled with those basement membranes.

The reduction of laminin in exfoliation-free vascular basement membranes may reflect an abnormal pattern of laminin utilisation. This suggests that laminin, which normally is incorporated into basement membrane, is being diverted to the formation of exfoliation material. This is not surprising, as the cells that produce the matrix components for the ocular basement membranes are probably the same as those proposed for the synthesis of exfoliation material. ${ }^{101314}$ The triggering mechanism behind this functional conversion awaits clarification, though a metabolic mechanism has been suggested. ${ }^{13} 14$

The absorption of laminin from the iris vascular basement membranes and the ensuing alterations of its binding with the other basement membrane constitutents, like HSPG and collagen type IV, could compromise the structural integrity and the filtration properties of iris vascular basement membranes. This underlying mechanism may account for the increased permeability of exfoliative iris vessels visualised by fluorescein angiography. ${ }^{34}$

Another point of clinical interest was the relationship between exfoliation material and the iris dilator muscle. In four exfoliative specimens there was extensive infiltration of the dilator muscle extracellular matrix with laminin labelled exfoliative fibres (Fig 2B). This phenomenon, together with a potential alteration of the laminin distribution in the embedding matrix of the dilator muscle, could account for the impaired dilatation commonly seen in patients with exfoliation syndrome and glaucoma. ${ }^{35}$

The adhesive properties of laminin are well documented. ${ }^{26} 27$ It may be instrumental in the adherence of exfoliation aggregates to ocular structures, such as the anterior hyaloid face and corneal endothelium.

The relationship between exfoliation material and ocular basement membranes has been the subject of fierce speculation over the last few decades. Ultrastructural studies have already documented a number of morphological features that support this relationship. ${ }^{36-40}$ These features in the iris comprise $(a)$ the proximity of exfoliation material to basement membranes, and $(b)$ the marked ultrastructural changes caused by exfoliation deposition on the iris vascular basement membranes. At a late stage these membranes may be almost entirely replaced by exfoliation material. ${ }^{39-41}$ This association became more apparent after a three-dimensional reconstruction study ${ }^{40}$ of exfoliation deposits and iris vessels; many stromal deposits were traced back to the vessel wall.

Despite the fact that most investigators in the field have favoured the involvement of basement membrane producing cells in the production of exfoliation material,,$^{131440}$ there is less agreement on whether or not exfoliation material contains basement membrane components. Evidence has already been advanced to indicate that exfoliation material contains glycoprotein ${ }^{37}$ and proteoglycans, ${ }^{46}$ which are major components of basement membranes. However, a number of investigations have repeatedly shown an association between exfoliation material and components of the elastic system, namely, oxytalan $^{89}$ and elastic-like materials. ${ }^{10-12}$ These components, with the possible exception of amyloid $P, 22$ are not thought to be normal basement membrane constituents. Nevertheless, on the grounds of the variations seen in the histochemical staining properties it is clear that exfoliation material is a composite of more than one substance. ${ }^{59}$ Thus it is likely that elastin and other constituents are produced as part of an aberrant extracellular matrix synthesis. This process is probably orchestrated by basement membrane producing cells. Our results suggest that, within the iris vasculature, exfoliation material is synthesised by contractile cells.

In conclusion, we hypothesise that the synthesis of exfoliation material is initiated at the molecular level by an aberrant basement membrane biosynthesis, which results in laminin being incorporated into the exfoliation material. The main challenge, however, remains the identification of this aberration as a first step towards prevention of this common blinding disorder.

We thank Shahida Abraham for expert technical assistance and Pat Bonnar for secretarial assistance. We are also grateful to Drs J $L$ Jay, J Dudgeon, G N Dutton, $R$ L Burton, and $H$ Hammer for the supply of specimens and facilitating our access to their patients. This study was supported by a grant from the W H Ross
Foundation ([Scotland). 
1 Forsius $\mathrm{H}$. Exfoliation syndrome in various ethnic populations. Acta Ophthalmol (Kbh) 1988; 66 (suppl): 71-85.

2 Jerndal T. Open angle glaucoma and the pseudo-exfoliation syndrome. In: Cairns JE, eds. Glaucoma. London: Grune and Stratton, 1986: 661-77.

3 Morrison JC, Green WR. Light microscopy of the exfoliation syndrome. Acta Ophthalmol (Kbh) 1988; 66: (suppl): 5-27.

4 Davanger M. Pseudo-exfoliation material. Electron microscopy after the application of lanthanum as tracer particles and ionic stain. Acta Ophthalmol (Kbh) 1980: 58: 512-9.

5 Ringvold A. Exfoliation syndrome. Immunological aspects. Acta Ophthalmol (Kbh) 1988; 66 (suppl): 35-43.

6 Harnisch JP, Barrach HJ, Hassel JR, Sinha PK. Identification of a basement membrane proteoglycan in exfoliation material. Graefes Arch Clin Exp Ophthalmol 1981; 215: 2738.

7 Streeten BW, Gibson SA, Li ZY. Lectin binding to pseudoexfoliative material and the ocular zonules. Invest Ophthalmol Vis Sci 1986; 27: 1516-21.

8 Garner A, Alexander RA. Pseudoexfoliative disease: histochemical evidence of an affinity with zonular fibres. $\mathrm{Br} \mathcal{F}$ Ophthalmol 1984; 68: 574-80.

9 Streeten BW, Dark AJ, Barnes CW. Pseudoexfoliative material and oxytalan fibres. Exp Eye Res 1984; 38: 523-31.

10 Streeten BW, Gibson SA, Dark AJ. Pseudoexfoliative material contains an elastic microfibrillar-associated glycoprotein. Trans Am Ophthalmol Soc 1986; 84: 304-20.

$11 \mathrm{Li} \mathrm{ZY}$, Streeten BW, Wallace RN. Association of elastin with pseudoexfoliative material: an immunoelectron microscopic study. Curr Eye Res 1988; 7 : 1163-72.

$12 \mathrm{Li} \mathrm{ZY}$, Streeten BW, Yohai N. Amyloid $\mathrm{P}$ protein in pseudoexfoliative fibrillopathy. Curr Eye Res 1989; 8: 21727.

13 Eagle RC, Font RL, Fine BS. The basement membrane exfoliation syndrome. Arch Ophthalmol 1979; 97: 510-5.

14 Dark AJ, Streeten BW. Pseudoexfoliation syndrome. In: Garner A, Klintworth G, eds. Pathobiology of ocular disease. A dynamic approach. New York: Dekker, 1982: 1303-20.

15 Marshall GE, Konstas AG, Lee WR. Immunogold localization of type IV collagen and laminin in the aging human aqueous outflow system. Exp Eye Res in press.

16 Marshall GE, Konstas AG, Lee WR. Ultrastructural distribution of collagen types I-VI in aging human retinal tribution of collagen types $1-\mathrm{V}$ in aging

17 Prince AM, Streeten BW, Ritch R, Dark AJ, Sperling M Preclinical diagnosis of pseudoexfoliation syndrome. Arch Ophthalmol 1987; 105: 1076-82.

18 Shakib M, Ashton N, Blach R. Electron microscopic study of pseudoexfoliation of the lens capsule. II. Iris and ciliary body. Invest Ophthalmol Vis Sci 1965; 4: 154-61.

19 Jay JL, Murray SB. Early trabeculectomy versus conventional management in primary open angle glaucoma. Brf Ophthalmol 1988; 72: 881-9.

20 Konstas AG, Marshall GE, Lee WR. Immunocytochemical localization of collagens (I-V) in the human iris. Graefes Arch Clin Exp Ophthalmol 1990; 228: 180-6.

21 Ben-Zvi A, Rodrigues MM, Krachmer JH, Fujikawa LS. Immunohistochemical characterization of extracellular matrix in the developing human cornea. Curr Eye Res 1986; 5: 105-17.
22 Abrahamson DR. Recent studies on the structure and pathology of basement membranes. F Pathol 1986; 149: 257-78.

23 Abbot NJ. The milieu is the message. Nature 1988; 332: 490-1.

24 Martin GR, Timpl R. Laminin and other basement membrane components. Ann Rev Cell Biol 1987; 3: 57-85.

25 Schnittny JC, Timpl R, Engel J. High resolution immunoelectron microscopic localization of functional domains of laminin, nidogen and heparan sulphate proteoglycan in epithelial basement membrane of mouse cornea reveals different topological orientations. F Cell Biol 1988; 107: 1599-610.

26 Engel J. EGF-like domains in extracellular matrix proteins: localized signals for growth and differentiation? FE BS Lett localized signals

27 Herbst TJ, McCarthy JB, Tsilibary EC, Furcht LT Differential effects of laminin, intact type IV collagen and specific domains of type IV collagen on endothelial cell adhesion and migration. F Cell Biol 1988; 106: 1365-73.

28 Panayotou G, End P, Aumailley M, Timpl R, Engel J. Domains of laminin with growth factor activity. Cell 1989; 56: 93-101.

29 Essner E, Lin WL. Immunocytochemical localization of laminin, type IV collagen and fibronectin in cat retinal laminin, type IV collagen and fibro

30 Grant DS, Leblond CP. Immunogold quantitation of laminin, type IV collagen and heparan sulphate proteoglycan in a variety of basement membranes. $\mathcal{f}$ Histochem Cytochem 1988; 36: 271-83.

31 Sugita A, Ishibashi R, Shiotani N, Yoshioka H. Morphological features of iris fibroblasts in dilator muscle region. $\mathcal{J p n ~} \mathfrak{F}$ Ophthalmol 1988; 32: 151-8.

32 Glaser BM. Extracellular modulating factors and the control of intraocular neovascularization. Arch Ophthalmol 1988; 106: 603-6.

33 Caldwell RB. Extracellular matrix alterations precede vascularization of the retinal pigment epithelium in dystophic rats. Curr Eye Res 1989; 8: 907-21.

34 Vannas A. Fluorescein angiography of the vessels of the iris in pseudoexfoliation of the lens capsule, capsular glaucoma and some other forms of glaucoma. Acta Ophthalmol (Kbh) 1969; 105 (suppl): 9-75.

35 Carpel EF. Pupillary dilatation in eyes with pseudoexfoliation syndrome. Am $\mathcal{f}$ Ophthalmol 1988; 105: 692-94.

36 Ringvold A. Light and electron microscopy of the wall of iris vessels in eyes with and without exfoliation syndrome. Virchows Arch $(A)$ 1970; 349: 1-9.

37 Ringvold A. The distribution of exfoliation material in the iris from eyes with exfoliation syndrome. Virchows Arch $(A)$ 1970; 351: 168-78.

38 Anastasi G, Puzzolo D, Romeo G, Santoro A, Scullica L. Iris ultrastructural changes in senile pseudoexfoliation. Ophthalmologica 1974; 168: 109-21.

39 Ghosh M, Speakman JS. The iris in senile exfoliation of the lens. Can f Ophthalmol 1974; 9: 289-97.

40 Shimizu T. Changes of iris vessels in capsular glaucoma: three dimensional and electron microscopic studies. $\mathfrak{f p}_{p n} \mathfrak{f}$ Ophthalmol 1985; 29: 434-52.

41 Dickson DH, Ramsey MS. Fibrillopathia epitheliocapsularis. Review of the nature and origin of pseudoexfoliative deposits. Trans Ophthalmol Soc UK 1979; 99: 284-92, 